“C 2010 IEEE. Personal use of this material is permitted. Permission from IEEE must be obtained for all other uses, in any current or future media, including reprinting/republishing this material for advertising or promotional purposes, creating new collective works, for resale or redistribution to servers or lists, or reuse of any copyrighted component of this work in other works.” 


\title{
Opportunistic Downlink Sum-Rate with Multiple Feedback Thresholds
}

\author{
K. Sandrasegaran and R. Patachaianand \\ Faculty of Engineering and Information Technology \\ University of Technology Sydney \\ Centre for Realtime Information Networks \\ 1 Broadway, Ultimo, New South Wales 2007, Australia \\ \{ kumbes@eng.uts.edu.au,rapatach@eng.uts.edu.au\}
}

\begin{abstract}
The achievable sum-rate capacity in multiuser systems grows as an increasing function of the number of users when opportunistic scheduling is employed. The scheduling requires the knowledge of channel state information (CSI) which is generated and sent from the users. It has been verified that the sum-rate capacity of a multiuser system with one-bit CSI feedback scheme by which each user whose channel quality is above an optimally selected threshold sends one-bit feedback has the same growth rate as that with the full CSI. However, the one-bit feedback scheme introduces non-negligible capacity loss to the achievable sum-rate. In this paper, a feedback reduction technique with multiple feedback thresholds is presented and analysed. The analysis yields closed-form expressions of the achievable sum-rate and its lower bound. The numerical results show that approximately $84 \%$ of the capacity loss can be eliminated in the system employing three optimally selected feedback thresholds. The lower-bound of the achievable sum-rate approaches the full-CSI sum-rate as the number of thresholds increases.
\end{abstract}

Keywords - Opportunistic scheduling, Multiuser systems, Limited feedback.

\section{INTRODUCTION}

A wireless communications system is typically designed to support a large number of users simultaneously. Opportunistic scheduling techniques by which only one user having the highest instantaneous channel quality in each scheduling block is selected to communicate with the base station can improve the overall capacity. This technique is referred to as multiuser diversity. To implement this idea, the scheduler must know which user to be scheduler in each scheduling block based on the users' channel quality. This information is sent, from users to the scheduler, by means of channel state information (CSI). Allowing all users to send feedback is waste of resources since there is at most one user to be scheduled in a scheduling block, and thus feedback sent from other users is waste of the resource.

Research work in recent years has presented several techniques to reduce feedback loads. One of the efficient techniques was presented and analysed in [1] and [2], where only users whose channel quality crosses a predefined threshold are allowed to send one-bit CSI. Mathematical analysis was given in [1] and [2] to prove that the capacity growth from multiuser diversity can be achieved using one-bit feedback (with single feedback threshold). Although the one-bit feedback technique can reduce feedback load significantly, it causes a loss in the system's achievable sum-rate. In [1] and [2], there is a nonnegligible capacity loss in the achievable capacity due to the use of the one-bit scheme. This calls for a technique to mitigate the capacity loss.

Various approaches have been proposed to minimise the amount of CSI feedback. In [3], a feedback reduction protocol where all users compete to send feedback via a share contention-based feedback channel is proposed. The idea of the contention-based feedback in [3] is to have a number of minislots in the uplink feedback channel for users to compete to send feedback messages. The users whose channel quality exceeds a threshold compete for one of the minislots with some predetermined probability. The same idea was extended for orthogonal frequency division multiple access (OFDMA) systems in [4]. The use of contention-based feedback was extended in [5] by dividing users into multiple groups each of which has a separate set of feedback minislots. In [6], an OFDMA resource allocation algorithm with multiple-threshold contentionbased feedback protocol was proposed. In [7], a contentionbased feedback protocol with multiple threshold in which users compete for feedback minislots based on their channel quality was introduced. In [8], an idea to minimise feedback employing multiple-threshold was proposed. With the feedback reduction algorithm in [8], the users are first probed with the highest threshold. If none of the users are above this threshold the threshold value is sequentially lowered until one or more users are found.

All work presented in [2]-[7], was proposed to minimise the amount of feedback minislots required to achieve near ideal case where full CSI is available to the transmitter. None of them attempted to minimise the actual amount of feedback sent from an individual user as considered in [1], [2] and [9]. However, techniques proposed in [1] and [2] result in capacity loss as discussed earlier (see also Fig. 3 in Section $\mathrm{V}$ in this paper). The algorithm proposed in [8] may also cause capacity loss if there is no user sending feedback within the feedback deadline and the amount of time to wait for at least one user sends feedback is not negligible.

This paper presents feedback schemes in which multiples feedback thresholds are applied to mitigate the capacity loss introduced by the use of one-bit feedback scheme. A closed-form expression of a system with an arbitrary 
number of feedback thresholds is proposed to represent the achievable sum-rate capacity of the proposed feedback techniques. The numerical results show that our proposed techniques can reduce the capacity loss caused by the one-bit feedback technique for all range of number of users. The proposed technique does not introduce any feedback delay as required in the threshold-based feedback proposed in [8].

This paper is organised as follows: The system model is explained in Section II. Section III describes the proposed threshold-based feedback protocol. Analysis of the proposed technique is discussed in Section IV. Numerical results are discussed in Section V. Section VI concludes this work.

\section{SYSTEM MODEL}

A single-carrier downlink multiuser system with single antenna at both receiver and transmitter where $K$ mobiles are communicating with a base station over a flatRayleigh fading channel is considered. At arbitrary time, the data transmission of the $k^{\text {th }}$ user can be modelled as

$$
y_{k}=h_{k} x+w_{k}
$$

where $y_{k}$ is the received signal, $h_{k}$ is the fading gain $h_{k} \sim$ $C \mathcal{N}(0,1)$, and $w_{k}$ is the additive white Gaussian noise $(\mathrm{AWGN}) x_{k} \sim \operatorname{CN}(0, \sigma)$. The normalised signal-to-noise ratio can then be computed by $\gamma_{k}=\left|h_{n}\right|^{2} / \sigma^{2}$. Given that the thermal noise power density $\sigma^{2}=1$, the probability density function (PDF) of $\gamma_{k}$ follows

$$
f_{\gamma_{k}}(x)=e^{-x} ; \quad x \geq 0 .
$$

Throughout this paper, the same average power constraint $P$ is assumed for all users.

Every mobile estimates its own channel quality in every scheduling block but only some mobiles transmit the channel quality information to the base station. In particular, only those whose channel quality is greater than the feedback thresholds send CSI.

\section{SUM-RATE WITH ONE FEEDBACK THRESHOLD}

Multiuser diversity gain can be obtained using one-bit CSI feedback. Somekh et al [2] analysed a multiuser system with an opportunistic scheduling. The base station (or the scheduler) broadcasts a feedback threshold $\alpha$ to all the users. Each user whose channel quality is above the threshold sends a CSI containing bit ' 1 ' to the transmitter. The transmitter randomly selects a user to be served based on the received one-bit information. In this system, the channel is active only if at least one user among $K$ users has the channel power gain above the threshold $\alpha$. The closed-form expression of the sum-rate, $R$, is given by [2]

$$
R(\alpha, K, P)=Q(\alpha, K) \cdot C(\alpha, P)
$$

where

$$
Q(\alpha, K)=\left(1-\left(1-e^{-\alpha}\right)^{K}\right)
$$

$$
C(\alpha, P)=\log (1+\alpha P)+e^{\left(\frac{1+\alpha P}{P}\right)} \cdot E i\left(\frac{1+\alpha P}{P}\right)
$$

$E i(x)=\int_{x}^{\infty} e^{-t} / t d t$ is the exponential integral function and $\log (x)$ is a natural logarithm of a variable $x . Q(\alpha, K)$ is the probability that at least one user has channel quality above the threshold $\alpha, \operatorname{Pr}\left(\gamma_{m}>\alpha\right)$ where $\gamma_{m}=\max _{k} \gamma_{k} \cdot C(\alpha, P)$ is referred to as the achievable ergodic rate of a scheduled user over the fading blocks,

$$
C(\alpha, P)=E_{\gamma_{\alpha}}\left\{\log \left(1+P \gamma_{\alpha}\right)\right\}
$$

where $E\{x\}$ is the expectation value of random variable $x$, and $\gamma_{\alpha} \in\left\{\gamma_{k} \mid \gamma_{k}>\alpha\right\} ; k=1, \ldots, K$.

The lower-bound capacity can be obtained by replacing the conditioned fading power $\gamma_{\alpha}$ in (5) by its lowest value $\alpha$ [2]. This yields the lower-bound capacity

$$
C_{L}(\alpha, P)=\log (1+P \cdot \alpha)
$$

\section{SUM-RATE WITH MULTIPLE FEEDBACK THRESHOLDS}

In this section, we propose feedback techniques with multiple thresholds and analyse the effect of the thresholds on the achievable sum-rate capacity of multiuser systems with opportunistic scheduling. The feedback bits are sent via reliable delay-free uplink channel, and the transmitter selects only one user to communicate to in each scheduling block period. The system with $N$ feedback thresholds and the corresponding lower bounds is shown in Figure 1.

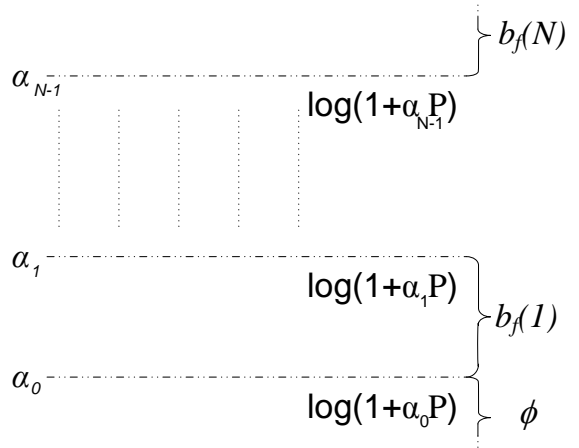

Fig. 1. System with $\mathrm{N}$ feedback thresholds

In Figure 1 , for $k^{\text {th }}$ user, $b_{f, k}(n)=\left\{b_{f}(0), b_{f}(1), \ldots, b_{f}(N)\right\}$ denotes the feedback bit(s) indicating the status of the scheduling metric if it is above the $n^{\text {th }}$ feedback threshold, otherwise no feedback is sent. For example, in a system with three feedback thresholds, $b_{f, k}$ may consists of two-bits and be described as follows

$$
b_{f, k}=\left\{\begin{array}{ll}
00 & ; \quad \gamma_{k}<\alpha_{0} \\
01 & ; \alpha_{0} \leq \gamma_{k}<\alpha_{1} \\
10 & ; \alpha_{1} \leq \gamma_{k}<\alpha_{2} \\
11 & ; \alpha_{2} \leq \gamma_{k}
\end{array} .\right.
$$


The transmitter receives the feedback bits sent from all the users and it randomly allocates all the transmission power to one of the users with the highest scheduling metric, i.e. $b_{f}(N)$. If there is no user sending $b_{f}(N)$, it allocates power to one of those sending $b_{f}(N-1), b_{f}(N-2)$, and so on. In the case if no feedback received, the transmitter keep silent for the current scheduling block period. Hence, the transmitter communicates with at most one user at all time. The achievable sum-rate of the system with $N \geq 2$ thresholds can be expressed by (see Appendix for proof)

$$
\begin{aligned}
R\left(\bar{\alpha}_{N}, K, P\right) & =Q\left(\alpha_{N-1}\right) \cdot C\left(\alpha_{N-1}\right) \\
& +\sum_{n=1}^{N-1}\left\{( Q ( \alpha _ { n - 1 } ) - Q ( \alpha _ { n } ) ) \cdot \left(C\left(\alpha_{n-1}\right)\right.\right. \\
& \left.\left.-\exp \left(-\delta_{n}\right) \cdot C\left(\alpha_{n}\right)\right) /\left(1-\exp \left(-\delta_{n}\right)\right)\right\}
\end{aligned}
$$

where $\bar{\alpha}_{N}$ is a $1 x N$ dimension vector whose elements are feedback thresholds, $\bar{\alpha}_{N}=\left\{\alpha_{0}, \ldots, \alpha_{N-1}\right\}$.The factor $\delta_{n}$ is defined as $\delta_{n}=\alpha_{n-1^{-}} \alpha_{n}$. K and $P$ are constant and omitted in this equation.

The lower bound of (9) can be expressed as (see Appendix for proof)

$$
\begin{aligned}
R_{L}\left(\bar{\alpha}_{N}, K, P\right) & =Q\left(\alpha_{N-1}\right) \cdot C\left(\alpha_{N-1}\right) \\
& +\sum_{n=1}^{N-1}\left\{\left(Q\left(\alpha_{n-1}\right)-Q\left(\alpha_{n}\right)\right) \cdot\left(C\left(\alpha_{n-1}\right)-C\left(\alpha_{n}\right)\right)\right\}
\end{aligned}
$$

The proposed multiple thresholds technique requires the receivers (i.e. mobile users) to compare the estimated channel quality with the thresholds and send CSI to the transmitter. Therefore, its computation complexity at mobile site is similar to the one-bit feedback scheme in [1] and [2]. At the transmitter, the optimum feedback thresholds can be computed in advance and broadcasted to all users. This can be performed offline, and thus no additional real-time complexity is required to implement the proposed scheme.

\section{NUMERICAL RESULTS}

In Figure 2, the achievable sum-rate and its lower bound are plotted versus the number of feedback thresholds when the number of users $K=200$.

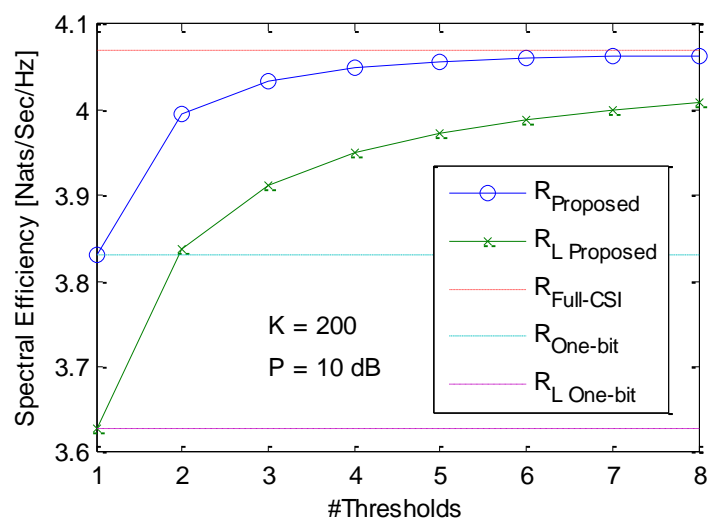

Fig. 2. Spectral efficiency versus number of thresholds
In Figure 2, $\mathrm{R}_{\text {Proposed }}$ represents the achievable sum-rate of the proposed feedback scheme with multiple thresholds, and $\mathrm{R}_{\mathrm{L} \text { Proposed }}$ represents its lower bound. This figure verifies that the achievable spectral efficiency and its lower bound increase as a function of the number of feedback thresholds. There is non-negligible sum-rate loss of around 0.237 Nats/sec/Hz for the one-bit feedback in [2], and 0.442 Nats/sec/Hz for its lower bound. The proposed feedback scheme with three thresholds can reduce the capacity loss to $0.036 \mathrm{Nats} / \mathrm{sec} / \mathrm{Hz}$ for the achievable sum-rate capacity and to $0.158 \mathrm{Nats} / \mathrm{sec} / \mathrm{Hz}$ for its lower bound. Provided that having more than three thresholds increase the capacity only slightly, and two-bit feedback is sufficient to represent all states of a system with three feedback thresholds, the threethreshold scheme is considered as an optimum.

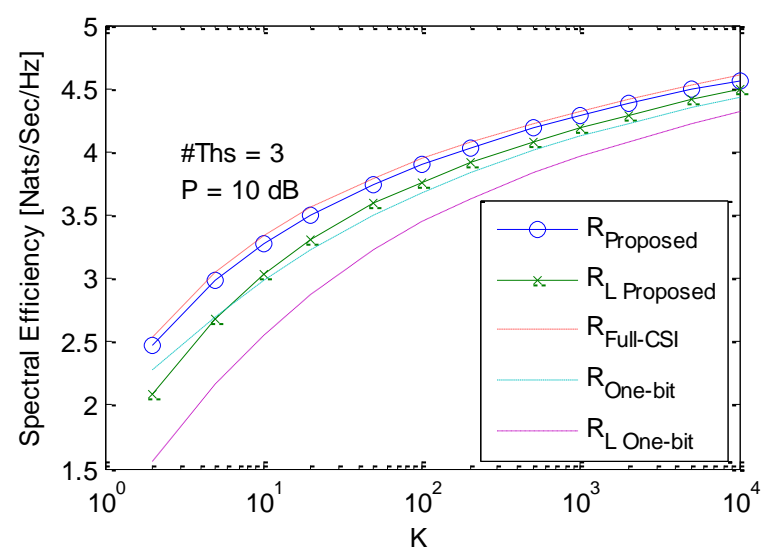

Fig. 3. Spectral efficiency versus number of users

Figure 3 shows the achievable sum-rate and its lower bound versus the number of users when three optimally selected feedback thresholds are employed. The optimum thresholds, $\dot{\alpha}$, for each $K$ can be calculated from heuristic search to satisfy:

$$
\alpha^{\prime}(K)=\underset{\bar{\alpha}}{\arg \max } R\left(\bar{\alpha}_{N}, K, P\right)
$$

Note that the optimum feedback thresholds can be precalculated for each given number of users. There is no need to calculate the optimum thresholds in real-time.

The capacity loss in achievable sum-rate $\left(\right.$ mean $_{\mathrm{k}}\left(\mathrm{R}_{\text {Full-CSI }}-\right.$ $\mathrm{R})$ for all $K$ ) reduces from $0.25 \mathrm{Nats} / \mathrm{Sec} / \mathrm{Hz}$ for the one-bit feedback scheme in [1] and [2] to $0.04 \mathrm{Nats} / \mathrm{Sec} / \mathrm{Hz}$ on average for the proposed three-threshold scheme. These numbers correspond to $84 \%$ reduction in capacity loss.

For the lower bound, the average capacity loss due to onebit feedback is as high as $0.54 \mathrm{Nats} / \mathrm{sec} / \mathrm{Hz}$, which is not negligible. The proposed three-threshold scheme can reduce the loss to $0.211 \mathrm{Nats} / \mathrm{Sec} / \mathrm{Hz}$, which correspond to $61 \%$ reduction. The loss can be further reduced by having more thresholds as shown in Figure 2. It is worth noting that the lower-bound capacity provides tighter bound from practical application since finite set of modulation and coding schemes are employed. 


\section{CONCLUSIONS}

In this work, we propose and analyse a multiuser system with opportunistic scheduling and multiple feedback thresholds. Closed-form expressions of the achievable sum-rate and its lower bound are presented. Numerical results show that the achievable sum-rate and its lower bound approach the full-CSI capacity when the number of optimally selected feedback thresholds increase. Three thresholds are sufficient to achieve near optimum $(<0.05 \mathrm{Nats} / \mathrm{sec} / \mathrm{Hz}$ average loss $)$ capacity. The three-threshold scheme reduces the loss in the lower bound by $61 \%$. This work can be applied with any contention-based mechanisms e.g. [3] - [8], and can be extended to OFDMA systems. This is leave for future work.

\section{APPENDIX \\ PROOF OF EQUATION 9 AND 10}

The achievable sum-rate of a multiuser system with $N$ feedback thresholds $\bar{\alpha}_{N}$ can be computed from

$$
\begin{aligned}
& R\left(\bar{\alpha}_{N}, K, P\right)=\operatorname{Pr}\left(\gamma_{m} \geq \alpha_{N}\right) \cdot E_{\gamma_{\alpha_{N}}}\left\{\log \left(1+P \cdot \gamma_{\alpha_{N}}\right)\right\} \\
& +\operatorname{Pr}\left(\alpha_{N}>\gamma_{m} \geq \alpha_{N-1}\right) \cdot E_{\gamma_{\alpha_{N}, \alpha_{N-1}}}\left\{\log \left(1+P \cdot \gamma_{\alpha_{N}, \alpha_{N-1}}\right)\right\} \\
& +\ldots+\operatorname{Pr}\left(\alpha_{1}>\gamma_{m} \geq \alpha_{0}\right) \cdot E_{\gamma_{\alpha_{1}, \alpha_{0}}}\left\{\log \left(1+P \cdot \gamma_{\alpha_{1}, \alpha_{0}}\right)\right\}
\end{aligned}
$$

where $\gamma_{\alpha_{N}, \alpha_{N-1}} \in\left\{\gamma_{k} \mid \alpha_{N}>\gamma_{k} \geq \alpha_{N-1}\right\}$.

Let us consider each term of RHS in (11) (K and P are constant and omitted). From (4), and (6) we have

$$
\operatorname{Pr}\left(\gamma_{m} \geq \alpha_{N}\right)=Q\left(\alpha_{N}\right)
$$

and

$$
E_{\gamma_{\alpha_{N}}}\left\{\log \left(1+P \cdot \gamma_{\alpha_{N}}\right)\right\}=C\left(\alpha_{N}\right) .
$$

Provided that $\operatorname{Pr}(A>x \geq B)=\operatorname{Pr}(B)-\operatorname{Pr}(A)$,

$$
\operatorname{Pr}\left(\alpha_{N}>\gamma_{m} \geq \alpha_{N-1}\right)=Q\left(\alpha_{n-1}\right)-Q\left(\alpha_{n}\right)
$$

Consider the expectation value term

$$
\begin{aligned}
E_{\gamma_{\alpha_{N}, \alpha_{N-1}}} & \left\{\log \left(1+P \cdot \gamma_{\alpha_{N}, \alpha_{N-1}}\right)\right\}= \\
& \int_{-\infty}^{\infty} \log (1+P \cdot x) \cdot f_{\gamma_{k}}\left(x \mid \alpha_{N}>x \geq \alpha_{N-1}\right) \cdot d x
\end{aligned}
$$

where $f_{\gamma_{k}}(x)$ is given in (2).

It is known that

$$
f_{\gamma_{k}}\left(x \mid \alpha_{N}>x \geq \alpha_{N-1}\right)=\left\{\begin{array}{lr}
0 & x<\alpha_{N-1} \\
\frac{f_{\gamma_{k}}(x)}{\alpha_{N}} f_{\gamma_{k}}(\chi) \cdot d \chi & \alpha_{N-1} \leq x<\alpha_{N} . \\
\int_{N-1} & \alpha_{N} \leq x
\end{array}\right.
$$

Applying (16) into (15) yields

$$
\begin{gathered}
E_{\gamma_{\alpha_{N}, \alpha_{N-1}}}\left\{\log \left(1+P \cdot \gamma_{\alpha_{N}, \alpha_{N-1}}\right)\right\}=\int_{\alpha_{N-1}}^{\alpha_{N}} \log (1+P \cdot x) \cdot \frac{e^{-x}}{e^{-\alpha_{0}}-e^{-\alpha_{1}}} \cdot d x \\
=\frac{1}{1-e^{-\left(\alpha_{N}-\alpha_{N-1}\right)}} \cdot\left(\int_{\alpha_{N-1}}^{\infty} \log (1+P \cdot x) \cdot e^{\alpha_{N-1}-x} \cdot d x\right. \\
\left.-e^{\alpha_{N-1}-\alpha_{N}} \int_{\alpha_{N}}^{\infty} \log (1+P \cdot x) \cdot e^{\alpha_{N}-x} \cdot d x\right)
\end{gathered}
$$

We know that

$$
C(\alpha, P)=E_{\gamma_{\alpha}}\left\{\log \left(1+P \gamma_{\alpha}\right)\right\}=\int_{\alpha}^{\infty} \log (1+P \cdot x) \cdot e^{\alpha-x} \cdot d x
$$

Applying (18) into (17) yields

$$
\begin{aligned}
\int_{\alpha_{N-1}}^{\alpha_{N}} \log (1+P \cdot x) & \frac{e^{-x}}{e^{-\alpha_{N-1}}-e^{-\alpha_{N}}} \cdot d x= \\
& \frac{1}{1-e^{-\left(\alpha_{N}-\alpha_{N-1}\right)}} \cdot\left(C\left(\alpha_{N-1}\right)-e^{-\left(\alpha_{N}-\alpha_{N-1}\right)} \cdot C\left(\alpha_{N}\right)\right) .
\end{aligned}
$$

Substituting (12), (13), (14), and (19) into (11), and applying $\delta_{n}=\alpha_{n-1^{-}} \alpha_{n}$ yields (9), which concludes this proof. Similar procedures are applicable for the lower bound in (10), excepted that $E_{\gamma_{\alpha_{N}, \alpha_{N}-1}}\left\{\log \left(1+P \cdot \alpha_{N}\right)\right\}=\log \left(1+P \cdot \alpha_{N}\right)$

\section{REFERENCES}

[1] S. Sanayei and A. Nosratinia, "Exploiting multiuser diversity with only 1-bit feedback," in Proc. IEEE Wireless Comm. and Net. Conf. 2005 (WCNC'05), Mar 2005, pp. 978 - 983.

[2] O. Somekh, A. M. Haimovich, and Y. Bar-Ness, "Sum rate analysis of downlink channels with 1-bit feedback," IEEE Commun. Letters, vol. 11, no.2, pp. 137-139, Feb, 2007.

[3] T. Tang and R. Heath, "Opportunistic feedback for downlink multiuser diversity," IEEE Comm. Letters, vol. 9, pp. 948950, Oct 2005.

[4] T. Tang, R. W. Heath, Jr., S. Cho, and S. Yun, "Opportunistic feedback in clustered OFDM systems", in proceedings of International Symposium on Wireless personal Multimedia Communications, San Diego, CA, Sep. 2006.

[5] S. Patil and G. de Veciana, "Feedback and opportunistic scheduling in wireless networks", IEEE Trans. Wireless Commun., vol. 6, no. 12, pp. 4227-4238, Dec. 2007.

[6] R. Agarwal, V. Majjigi, Z. Han, R. Vannithamby, and J. Cioffi, "Low complexity resource allocation with opportunistic Feedback over Downlink OFDMA Networks", IEEE JSAC Special Issue on Limited Feedback, Vol. 26, No. 8, Oct. 2008, pp. 1462-1472

[7] S. Jaewoo and J.M Cioffi, "Feedback reduction scheme for downlink multiuser diversity", IEEE Trans. Wireless Commun., vol. 8, no. 2, pp. 668-672, Feb. 2009

[8] V. Hassel, M.-S. Alouini, D. Gesbert, G.E Oien, G.E., "Exploiting multiuser diversity using multiple feedback thresholds", Vehicular Technology Conference, Stockholm, Sweden, pp. 1302 - 1306, 30 May-1 June 2005. 